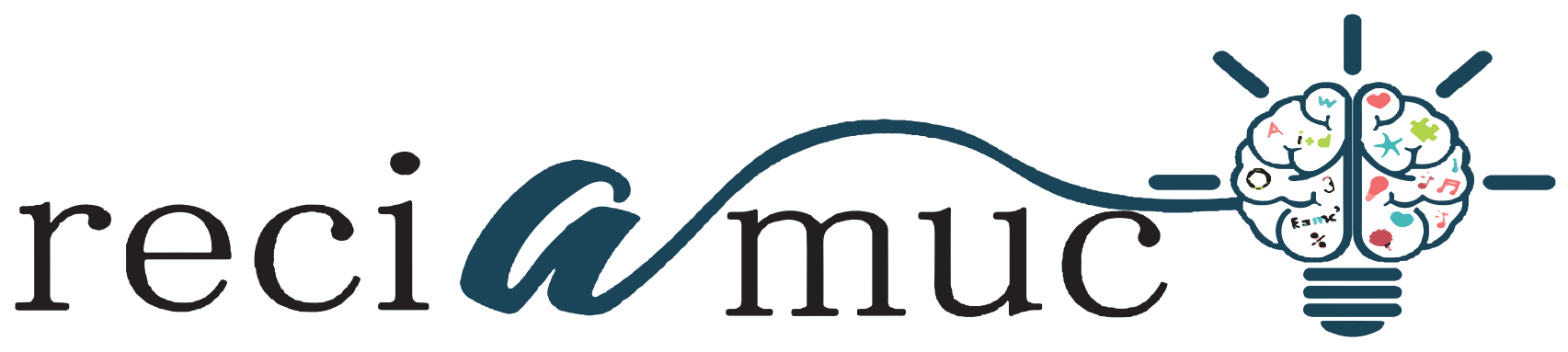

DOI: 10.26820/reciamuc/5.(1).ene.2021.416-429

URL: https://reciamuc.com/index.php/RECIAMUC/article/view/630

EDITORIAL: Saberes del Conocimiento

REVISTA: RECIAMUC

ISSN: 2588-0748

TIPO DE INVESTIGACIÓN: Artículo de investigación CÓDIGO UNESCO: 5801 Teoría y Métodos Educativos

PAGINAS: 416-429

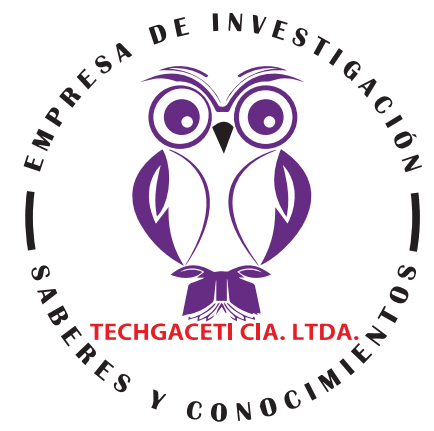

\title{
Estilos de crianza ante la violencia infantil
}

Parenting styles in the face of violence against children

Estilos parentais em face da violência infantil

\section{María Luisa Merchán Gavilánez'; Victoria María Márquez Allauca2; Jorge Fabian Yanez Palacios ${ }^{3}$; Lidia Patricia Estrella Acencio ${ }^{4}$}

\author{
RECIBIDO: 10/11/2020 ACEPTADO: 29/11/2020 PUBLICADO: 31/01/2021
}

1. Magister en Desarrollo de la Inteligencia y Educación; Especialista en Diagnostico Intelectual; Diploma Superior en Pedagogías Innovadoras; Licenciado en Ciencias de la Educación en la Especialidad de Supervisión y Administración Educativas; Profesor de Segunda Enseñanza en la Especialidad de Supervisión y Administración Educativas; Doctora en Ciencias de la Educación Especialización Gerencia Educativa; Universidad de Guayaquil; Guayaquil, Ecuador; maria.merchanga@ug.edu.ec; https://orcid.org/0000-0003-4971-0831

2. Magister en Terapia Familiar Sistémica y de Pareja; Diploma Superior en Terapia Familiar Sistémica; Psicóloga Clínica; Universidad de Guayaquil; Guayaquil, Ecuador; victoria.marqueza@ug.ed.ec; https://orcid.org/0000-0001-9093-551X

3. Diplomado en Educación Superior; Magister en Tecnología de la Información y Multimedia Educativa; Ingeniero en Sistemas; Licenciado en Administración de Empresas; Universidad de Guayaquil; Guayaquil, Ecuador; jorge.yanezp@ug.edu.ec; https://orcid.org/0000-0002-4727-1794

4. Magister en Educación Parvulario; Especialista en Educación Inicial; Licenciada en Educación Parvulario; Educador de Párvulos; Universidad de Guayaquil; Guayaquil, Ecuador; lidia.estrellaa@ug.edu.ec; https://orcid.org/0000-00033628-0481

\author{
CORRESPONDENCIA \\ María Luisa Merchán Gavilánez \\ maria.merchanga@ug.edu.ec \\ Guayaquil, Ecuador
}




\section{RESUMEN}

Los estilos de crianza constituyen una constelación de actitudes hacia el niño, que le son comunicadas creando un clima emocional en el que se ponen de manifiesto los comportamientos de los padres para educar y proteger a sus hijos. Cuando se asume un estilo de crianza democrático se establece claramente las reglas de comportamiento, prácticas afectivas y se disminuye las actitudes violentas. Se realizó un estudio documental bibliográfico en bases de datos: Scopus, Redalyc, ResearchGate y Scielo, acerca de los estilos de crianza, competencias parentales, carencias afectivas, factores psicosociales y estadísticas de violencia infantil. Entre los hallazgos más importantes se encontró que en Ecuador el $47 \%$ de los niños, niñas y adolescentes han recibido -por parte de sus progenitores- algún tipo de maltrato físico. De esa cifra, el $48 \%$ vivió castigos extremos (golpes, encierros, baños en agua fría y privación del alimento). El 75\% de la violencia proviene del padre, madre o de ambos progenitores. El estilo de crianza más común es el autoritario y permisivo. Estudios realizados en España, Estados Unidos destacan que los factores de ansiedad, estrés, depresión, déficit de atención de las madres influyen en la manera de educar a sus hijos o tutoriados. Los cuidadores habituales que no desarrollan competencias de parentalidad positiva probablemente continuarán con estilos de crianza basado en la violencia, permisividad o negligencia.

Palabras clave: Crianza disfuncional, parentalidad positiva, violencia infantil.

\section{ABSTRACT}

Parenting styles constitute a constellation of attitudes towards the child, which are communicated to them creating an emotional climate in which the behaviors of parents to educate and protect their children are revealed. When a democratic parenting style is assumed, the rules of behavior, affective practices are clearly established and violent attitudes are reduced. A bibliographic documentary study was carried out in databases: Scopus, Redalyc, ResearchGate and Scielo, about parenting styles, parental skills, affective deficiencies, psychosocial factors and statistics of childhood violence. Among the most important findings, it was found that $47 \%$ of children and adolescents in Ecuador have received some form of physical abuse from their parents. Of this number, $48 \%$ lived extreme punishments (beatings, confinements, baths in cold water and deprivation of food). $75 \%$ of the violence comes from the father, mother or both parents. The most common parenting style is authoritative and permissive. Studies carried out in Spain, the United States highlight that the factors of anxiety, stress, depression, attention deficit of mothers influence the way of educating their children or tutors. Regular caregivers who do not develop positive parenting skills will likely continue parenting styles based on violence, permissiveness, or neglect.

KeyWords: Dysfunctional parenting, positive parenting, child violence.

\section{RESUMO}

Os estilos parentais constituem uma constelação de atitudes em relação à criança, que lhes são comunicadas criando um clima emocional no qual os comportamentos dos pais para educar e proteger seus filhos são revelados. Quando um estilo parental democrático é assumido, as regras de comportamento, as práticas afetivas são claramente estabelecidas e as atitudes violentas são reduzidas. Um estudo documental bibliográfico foi realizado em bancos de dados: Scopus, Redalyc, ResearchGate e Scielo, sobre estilos parentais, habilidades parentais, deficiências afetivas, fatores psicossociais e estatísticas de violência infantil. Entre as descobertas mais importantes, constatou-se que $47 \%$ das crianças e adolescentes no Equador receberam alguma forma de abuso físico de seus pais. Desse número, $48 \%$ viveram castigos extremos (espancamentos, confinamentos, banhos em água fria e privação de alimentos). $75 \%$ da violência vem do pai, da mãe ou de ambos os pais. O estilo mais comum de paternidade é autoritário e permissivo. Estudos realizados na Espanha, os Estados Unidos destacam que os fatores de ansiedade, estresse, depressão, déficit de atenção das mães influenciam a forma de educar seus filhos ou tutores. Os cuidadores regulares que não desenvolvem habilidades parentais positivas provavelmente continuarão estilos parentais baseados na violência, permissividade ou negligência.

Palavras-chave: Parentalidade disfuncional, paternidade positiva, violência infantil. 


\section{Introducción}

En la sociedad actual se dan algunas situaciones que pueden vulnerar los derechos de niñas, niños y adolescentes en el contexto educativo, familiar, afectivo y sociocultural, ante lo cual el grupo más desprotegido es la niñez debido a que tienen pocos recursos para afrontar y defenderse ante las situaciones de maltrato y violencia que se encuentra en su entorno.

Se define el maltrato infantil como los abusos y la desatención de que son objeto los menores de 18 años, e incluye todos los tipos de maltrato físico o psicológico, abuso sexual, desatención, negligencia y explotación comercial o de otro tipo que causen o puedan causar un daño a la salud, desarrollo o dignidad del niño, o poner en peligro su supervivencia, en el contexto de una relación de responsabilidad, confianza o poder, la exposición a la violencia de pareja también se incluye a veces entre las formas de maltrato infantil. (OMS, 2016). Definitivamente, el maltrato tiene muchas formas de expresión y cualquiera de ellas siempre va junto con una o más de las otras, de acuerdo a los diversos contextos (Gabarino y Kostelny 1993 p. 213), otro aspecto que se debe tomar en cuenta es el abuso emocional el cual implica causar daño por acción u omisión de manera que se deteriora su funcionamiento, además puede incluir el rechazo, terror, aislamiento, explotación, degradación, ridiculización o corrupción. El Observatorio Social del Ecuador en el año 2010, menciona que el $44 \%$ de los niños y niñas de entre 5 y 17 años había sido objeto de respuestas violentas por parte de sus padres en el hogar ante el cometimiento de alguna falta o no obediencia", entre uno de los objetivos del PNBV estaba el "consolidar la transformación de la justicia y fortalecer la seguridad integral, en estricto respeto de los derechos humanos" e incluía políticas para erradicar la violencia de género y la construcción de una cultura de paz social y convivencia ciudadana, otro dato estadístico en el contexto ecuatoriano, no sólo la niñez sino también las mujeres son vulneradas y maltratadas el $50 \%$ de estas fueron víctimas de violencia en alguna de sus formas; la Encuesta Nacional de Relaciones Familiares y Violencia de Género en contra de las Mujeres, realizada por el INEC entre noviembre y diciembre del 2011, determinó que seis de cada 10 mujeres fueron víctimas de violencia, siendo Bolívar la provincia con el mayor porcentaje de casos.

La violencia se presenta en diferentes contextos familiares, sociales, escolares pero en el presente artículo queremos resaltar la violencia que se presenta en el entorno familiar. Niñas y niños son considerados población vulnerable, quienes reciben protección desde las políticas públicas, es decir, las políticas de protección de la niñez y adolescencia son acogidas por entidades gubernamentales y no gubernamentales, sin embargo las familias desconocen los derechos que tienen los niños.

La familia necesita desarrollar competencias parentales que les permitan educar, orientar y guiar a sus miembros que están en proceso de desarrollo psicológico, socioafectivo y emocional. Según Barudy y Dantagnan consideran que las competencias parentales son este saber hacer "las capacidades prácticas que tienen las madres y los padres para cuidar, proteger y educar a sus hijos, y asegurarles un desarrollo suficientemente sano. Las competencias parentales forman parte de lo que hemos llamado la parentalidad social, para diferenciarla de la parentalidad biológica, es decir, de la capacidad de procrear o dar la vida a una cría". Barudy hace énfasis en que las competencias son capacidades adquiridas por los padres y madres para la crianza de sus hijos, sin embargo habría que tomar en cuenta algunos otros aspectos como: tipología familiar, la diversificación del trabajo, el rol activo de la mujer como proveedora de la economía familiar, por lo tanto los hijos se quedan a cargo de cuidadores habituales sean estos familiares o no quienes tendrían que desarrollar estas competencias parentales. 
Mientras que Bronfenbrenner \& Evans determinan que la "competencia", guía el ejercicio parental a través de diversas situaciones de la vida familiar y propias de la crianza para garantizar el bienestar y el ejercicio pleno de los derechos de niñez y adolescencia.

En la investigación realizada en España por Limi Ana, Suria Raquel, acerca de los problemas de conducta infantil y competencias parentales en contextos de violencia, se encontró que un $63,6 \%$ de los y las hijas de las víctimas presenciaron o escucharon alguna de las situaciones de violencia, y el $64,2 \%$ sufrieron a su vez violencia, esto junto con numerosos trabajos visibilizan el impacto que tienen en niñas, niños y adolescentes, la exposición a la violencia de género.

De acuerdo a este estudio se puede relacionar que las consecuencias del maltrato hacia la mujer afectan el desempeño de la misma en su rol de madre, espacialmente en las respuestas afectivas, de satisfacción de las necesidades básicas y por ende tienen a maltratar a sus hijos.

Los cuidadores habituales que no desarroIlan competencias de parentalidad positiva probablemente continuarán con estilos de crianza basado en la violencia, permisividad o negligencia. Los estilos de crianza inadecuados pueden provocar carencias afectivas y formas de violencia normalizada en los niños en etapa escolar. El propósito del presente ensayo es el análisis documental bibliográfico de los estilos de crianza, competencias parentales, y formas de violencia.

Los propósitos del siguiente artículo son:

- Aproximación bibliográfica y documental a los términos de estilos de crianza y violencia infantil mediante la revisión de artículos científicos de los últimos años.

- Caracterizar los estilos de crianza desde el punto de vista de diferentes autores.

- Identificar resultados de investigaciones acerca de factores que influyen en los estilos de crianza y estadísticas de violencia infantil.

\section{Desarrollo}

\section{Métodos y materiales}

Se utiliza el paradigma cualitativo mediante el análisis documental de los estilos de crianza, violencia infantil, carencias afectivas, y competencias parentales, se realiza la revisión de 80 artículos científicos publicados en los últimos años, en las bases de datos de Scopus, Redalyc, ResearchGate y Scielo, así como planes, programas para la prevención de la violencia. El Análisis Documental se utilizó para el estudio interno del contenido de los artículos publicados acerca de las variables en estudio con el propósito de extraer las conceptualizaciones, características, clasificaciones, estadísticas y términos que definen el contenido. Esto permite recuperar información relevante y actualizada. Para el registro de información se diseña una ficha técnica en la cual se ubicaron datos relevantes extraídos del resumen, fundamentos teóricos, resultados y conclusiones de los artículos publicados. Se representa en diagramas semánticos el análisis de términos, características, tipologías, factores con el propósito de diagramar en forma de red las coincidencias, diferencias, clasificación, conceptuales de los estilos de crianza y la violencia infantil.

\section{Bases teóricas}

Se realizó una exhaustiva investigación documental acerca de los estilos de crianza, competencias parentales, violencia. Para hablar sobre los estilos de crianza, nos referimos a un conjunto de conductas o competencias aplicadas por los cuidadores o padres hacia los hijos o protegidos. Los padres o adultos a cargo son los principales responsables del cuidado, y protección y formación de los niños, desde la infancia hasta la adolescencia (Céspedes, 2008; Papalia, 2005; Sordo, 2009). Estas competencias parentales facilitan un clima

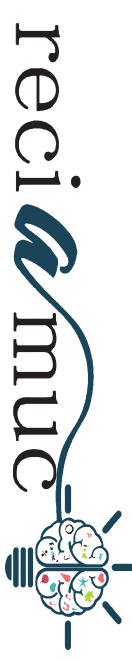


emocional favorable para el desarrollo del comportamiento y vínculo afectivo entre los cuidadores y los protegidos. Esto hace referencia a que los padres o tutores responsables del cuidado, así como de la enseñanza en cuanto al conocimiento de reglas, valores, roles, hábitos necesarios, etc. para que a medida de su desarrollo los niños y futuros adolescentes puedan desenvolverse de manera independiente y dentro de los preceptos adecuados en la sociedad actual. (Gomez, 2014)

Para Pérez \& Cánovas, 1996, p.141. "Son modelos o esquemas prácticos que simplifican las pautas de crianza y educación paterna en determinadas dimensiones básicas que, cruzadas entre sí en diferentes condiciones, dan lugar a diversos y habituales tipos de educación familiar. las prácticas de crianza se encuentran condicionadas por la personalidad, experiencias, características genéticas, factores socioeconómicos, la cultura que interactúan en el microsistema para educar a los hijos o protegidos para su bienestar integral.

Una de las principales investigaciones de Baumrind y por la cual surgen los estilos de crianza parental fue en sus estudios de 1966,1967 y 1971 sobre los efectos de los diferentes tipos de padres sobre la forma de crianza de un niño, observo tres grupos de niños preescolares.

Sus resultados fueron congruentes y demostró que los preescolares desconfiados e infelices tenían padres controladores y poco afectuosos. Los preescolares autosuficientes y felices tenían padres exigentes pero comunicativos. Los inmaduros y dependientes tenían padres cálidos que no fijaban límites. Sobre esta base, Baumrind desarrolló los Estilos de Crianza Autoritarios, Democráticos y Permisivos (Flinn, 2013). Según García y Becker en 1996, la investigación sobre estilos parentales y desarrollo personal y social del niño, demuestran que existen dos componentes principales; la identificación afectiva y el control comportamental.
Entonces se puede deducir que para que la crianza sea efectiva es primordial considerar los comportamientos afectivos entre padres e hijos, pero a la vez el control, para encauzar pautas, valores, normas, límites que serán respetados por los hijos pero a la vez que sean parte del comportamiento parental. En síntesis es indispensable una buena comunicación y socialización. Según Molpeceres, Musitu y Lila, 1994 el "afecto" a su vez se subdivide en dos dimensiones bipolares las cuales caracterizan las relaciones entre padres e hijos y son:

a) Aceptación- rechazo: dimensión que oscila entre el afecto, cariño parental, expresión de sentimientos positivos hacia la conducta del niño y por otra parte las expresiones físicas o verbales de agresividad, hostilidad o indiferencia.

b) Sobreprotección- distanciamiento: existen familias en donde su forma de actuar, su modo de vida o incluso su entorno hace que los miembros de esta familia este siempre junta, existen también familias desligadas donde sus miembros aparecen distanciados, sin comunicación fluida y no manifiestan una identidad común.

Al momento de hablar acerca de la crianza de los niños se debe tener en cuenta que ninguna familia en lo absoluto va a ser igual a otra, o van a tener los mismos patrones de crianza, todo varia debido a que dentro del ambiente familiar contamos con factores que influyen directamente en la formación de los niños como son el carácter del padre ,temperamento de la madre, el nivel de instrucción, estrato socioeconómico, nivel de madurez de los padres, tipos de familia entre otras influencias externas.

Según el libro Pueblos Indígenas y Educación No 59 (2006): Se presentan las definiciones de los métodos disciplinarios o estilos de crianza que se presentan en el presente trabajo: 
Estilo Permisivo.- Este tipo de disciplina es utilizada por padres que son sumamente cuidadosos por no herir a sus hijos e hijas. Caracterizado por la tendencia a manifestarle ternura a un nivel alto y menos control paterno.

Estilo Autoritario.- Se caracteriza por utilizar una disciplina drástica, rígida e inflexible, considerando que lo más relevante es el cumplimiento de las normas, del reglamento, del orden y la preservación de la autoridad.

Estilo Negligente.- Los padres muestran desinterés en la educación, bienestar emocional y físico de sus hijos. Están ausentes aunque estén presentes físicamente, no satisfacen necesidades del niño. Se caracteriza por ausencia de consejería, orientación, asesoramiento, expresión de afecto y falta de crítica.

Estilo Democrático.- Uno de los puntos que distingue la crianza democrática es el equilibrio entre la necesidad del padre de ejercer el control y su receptividad a la necesidad del chico de ser más independiente y responsable.

En la actualidad el estilo de crianza más común es el autoritario y permisivo llegando a extremos que causan daños en los niños tanto como el castigo, así como el amor exagerado que se le puede brindar, o también como el desinterés total, para poder comprender cuales son los problemas debemos tener en cuenta primero que al llegar a la utilización exagerada de una situación ya es un problema como los castigos excesivos, la muestra de afecto y el dejar que el niño se desenvuelva solo en forma permanente es un daño para los niños por lo que se debe buscar un punto medio entre estos tres estilos de crianza. Considerando que en este escenario sea muy común las carencias afectivas.

En la mayoría de los casos los padres o cuidadores trabajan todos el día permaneciendo los hijos a cargo de terceros, por otro lado el avance tecnológico a bloqueado la relación interpersonal entre los miembros de la familia, pues normalmente todos tienen sus celulares encendidos, y los más pequeños quedan en segundo plano.

Las responsabilidades que tienen tanto el padre como la madre con sus obligaciones en el hogar y trabajo, buscan la forma más rápida de hacer de sus hijos niños responsables en la escuela y el hogar, para esto en su mayoría se puede hablar que han optado por utilizar la violencia como forma de disciplina, día a día es utilizada por más familias, pero con el afán de que este método obtenga resultados visibles e incluso en su pensamiento se podría decir que buscan resultados inmediatos lo utilizan con frecuencia y con intensidad, y que al final podemos ya hablar de abuso físico en los niños, daños físicos a simple vista pero analizando encontraremos daños más severos como son los psicológicos, aun cuando los padres crean que al utilizar este método están obteniendo el resultado deseado.

Existen factores de personalidad, temperamento, contexto social, trastornos psicológicos de los padres o cuidadores que influyen en los estilos de crianza así lo demuestran investigaciones realizadas por Arellano en el 2018, quien realizó un estudio "Reacciones de los padres al afecto negativo de los niños. El papel moderador de los padres" Los resultados indican que GAD (trastornos de ansiedad generalizada) fortalece la relación entre el afecto negativo y el desánimo emocional, y que los padres con GAD no ajustan sus niveles de control para satisfacer las necesidades del niño. Estos hallazgos resaltan la importancia de enseñar a los padres con herramientas eficaces de GAD para manejar las emociones. Además, los tratamientos para padres con TAG pueden beneficiarse al abordar específicamente las interacciones con los niños y proporcionarles a los padres formas efectivas de enseñar a sus hijos a manejar y expresar emociones. (Arellano et al., 2018) 
Los factores de riesgo de coerción como la hostilidad materna, síntomas depresivos maternos, problemas de externalización infantil y negatividad diádica se asocian con la rigidez o inconsistencia en la crianza de los hijos al dar una respuesta al mal comportamiento de riesgo de coerción en los patrones de crianza. Las díadas madre-hijo. el riesgo combinado de los padres en general (es decir, rigidez) junto con cambios hacia una mayor variabilidad factores de riesgo confieren rigidez en la crianza de los hijos en general, pero en los los resultados mixtos anteriores y ofrecer nuevas direcciones para las intervenciones 303- 315)

Se realizó el estudio de las contribuciones de los síntomas de trastorno de déficit de atención materno, trastorno de oposición desafiante a la crianza de los hijos, síntomas depresivos, comportamiento parental, así como los comportamientos agresivos de sus adolescentes. Participaron 107 madres. Se concluyó que los comportamientos de trastorno de oposición desafiante materno se asociaron particularmente con las dimensiones negativas y afectivas de los padres.

Las investigaciones realizadas por Johnston, Miller, Mash y Ninowski, (2012) acerca de las posibles conexiones entre las características del TDAH en adultos y la crianza interrumpida. se determinó que los síntomas de falta de atención e impulsividad pueden afectar la capacidad de los padres para rastrear el comportamiento del niño, respondan con calma y coherencia a sus hijos, ser consistente. Los problemas en la autorregulación y la motivación también pueden dificultar que los padres con TDAH controlen su afecto, disciplina inconsistente, y bajos niveles de monitoreo infantil. Esto implica que el trastorno TDAH, depresión materna y conductas de externalización de las madres inciden en la crianza de sus hijos porque pueden verse abrumados por el comportamiento del niño.(Dudek et al., 2020)
Kohlhoff (2016) examinó las estrategias cognitivo-afectivas utilizadas por los padres de niños pequeños con problemas de conducta para regular las emociones. Las asociaciones entre la estrategia de regulación de las emociones y la calidad de la crianza de los hijos fueron moderadas por la severidad de los síntomas depresivos de los padres, con efectos distintos en las prácticas de crianza positivas y negativas. En términos de crianza positiva, el uso más frecuente de la reevaluación cognitiva se asoció con un mayor uso de elogios etiquetados entre los padres con niveles más bajos de síntomas depresivos, mientras que los padres que tenían niveles más altos de síntomas depresivos participaron en menos elogios, independientemente de la frecuencia con la que utilizaron la reevaluación. Las Tácticas de control aversivas como hablar duro o críticamente al niño, refuerzan las interacciones coercitivas entre padres e hijos así la excitación y angustia emocional negativa están asociadas con prácticas negativas de crianza. Los hallazgos más importantes de este estudio se encontró que los padres que participaban en altos niveles de reevaluación cognitiva eran los que tenían más probabilidades de brindar a sus hijos elogios efectivos como refuerzo positivo para el comportamiento deseable por lo tanto sugieren que las intervenciones destinadas a aumentar la crianza positiva y disminuir la crianza negativa pueden mejorarse aún más mediante la incorporación de estrategias ER siendo beneficioso para las familias de padres con depresión o que no tienen problemas de humor: la reevaluación apoya el crecimiento en la crianza positiva. (Kohlhoff et al., 2016, pp. $303-315$ )

De acuerdo a las investigaciones realizadas: Los diferentes factores psicosociales, trastornos, ansiedad, depresión, déficit de atención con hiperactividad, la motivación , autorregulación de los padres o cuidadores inciden en estilos de crianza Resulta interesante identificar que existen otros estilos de crianza según los factores de influencia, 
como: La crianza interrumpida debido al déficit de atención de uno de sus padres tienen dificultad para monitorear y hacer seguimiento al comportamiento del niño, no responden con calma y coherencia a sus hijo: La crianza consistente basada en elogios efectivos.

Se encontraron hallazgos interesantes en la investigación realizada a sesenta y seis mujeres con antecedentes de depresión mayor fueron seguidas durante el embarazo, el posparto y observadas durante las interacciones de juego y alimentación con sus bebés de 12 meses. Los hallazgos de la investigación surgen de que la prevención de la recurrencia de la depresión durante el embarazo puede tener el beneficio adicional de la participación de las mujeres en una crianza sensible; en otro trabajo se encontró que la depresión perinatal persistente, los padres no se involucran con su bebé a la que se llama crianza insensible que están relacionadas con las características personales, contextuales y el temperamento infantil. (Goodman et al., 2017, pp. 31 -47) La depresión y la ansiedad pueden interferir en la forma criar a los hijos entonces se deduce que los estilos de crianza que adoptan los padres de manera consciente o inconsciente se pueden relacionar con factor psicológicos de ansiedad y depresión ocasionando algún tipo de maltrato infantil físico, psicológico, desatención y negligencia.

La desconexión moral es un fuerte predictor de comportamiento agresivo, incluido el acoso escolar. Se examinó la relación entre las prácticas de crianza deficientes específicas y la desconexión moral estaba mediada por la aprobación esperada de los padres de la agresión y si la edad era un moderador en un modelo de mediación. En la adolescencia temprana, los niveles más bajos de monitoreo y supervisión se asociaron con niveles más altos de desconexión moral un año después, Estos resultados demuestran que en la infancia tardía y la adolescencia temprana, las prácticas de crianza deficientes, una disciplina más estricta y un control deficiente, son relevantes para la desconexión moral, en parte porque crean la expectativa de la aprobación de los padres para la agresión.(Campaert et al., 2018) .Según el estudio resalta la crianza deficiente en la moralización del niño y adolescente por la escaso seguimiento, monitoreo e involucramiento en la vida de sus hijos o tutorados.

Ante estos factores que influyen directamente en la forma o método de disciplinar a los niños y adolescentes, surge la violencia normalizada puesto que se tiene interiorizado que los niños son propiedad de los padres y que se debe criar a los hijos como fueron criados. (gritos, golpes, humillaciones, prohibiciones). Se ha normalizado el maltrato infantil a tal punto que se cree que, si son castigados, humillados, será beneficioso para que sean buenas personas.

Es muy común observar que los padres o cuidadores utilizan los malos tratos como formas de disciplinar y educar a sus hijos o protegidos, puesto que piensan que es la manera de evitar que se nieguen a obedecer o con la falsa creencia que los padres son los que mandan en la casa. En este ensayo abordaremos como los estilos de crianza puede conllevar algún tipo de violencia en contra de los niños y niñas.

Por violencia se entiende cualquier acción u omisión intencional que daña o puede dañar a un individuo y que en último extremo, perturba o restringe su capacidad para diseñar la vida en libertad. Los derechos humanos tratan de garantizar el ejercicio de esta capacidad. En consecuencia, una teoría de la justicia basada en los derechos humanos ha de considerar injusta cualquier forma de violencia. (Esplugues 2008).

La violencia se define como "comportamiento violento, amenazante u otro comportamiento de una persona que coacciona o controla a un miembro de la familia de la persona o hace que el miembro de la familia tenga miedo".(Douglas \& Nagesh, 2019). 
La legislación enumera varios ejemplos de comportamiento que pueden constituir violencia familiar, destacando que DFV no se limita al abuso físico, sino que también puede incluir el abuso emocional, económico, verbal y espiritual / cultural. Los dos autores coinciden que son acciones para someter u obligar a otra persona hacer algo, utilizando actos violentos, discriminatorios, humillaciones y abusos. En los estilos de crianza como la manera de educar a los niños o adolescentes surgen métodos disciplinarios dóciles, severos, indiferentes y negligentes debido a diferentes factores personales, contextuales y psicológicos que influyen en la manera de actuar de los padres o personas que están al cuidado de los menores. Reyes (2004) propone la tipología de la violencia infantil:

Violencia física: relacionada con el uso de la fuerza humana para deteriorar las condiciones de otro ser, objeto o sí mismo. Abarca golpes, bofetadas, empujones, patadas, heridas por armas de fuego o blancas, y todas aquellas acciones que van en detrimento de la vida.

Violencia verbal o emocional: incluyen todos los actos en los que una persona lastima psicológica o emocionalmente a otra, a través de gritos, desprecios, agresiones, insultos, mentiras, no respeto a la privacidad, a sus creencias e ideas, comentarios sarcásticos y burlas que exponen a la víctima al público, incluye expresiones como "eres un inútil o no sirves para nada".

Violencia sexual: abarca ataques sexuales directos como violaciones, hasta la incitación a realizar actos de índole sexual sin el consentimiento de la otra persona, pasando por la violencia verbal dirigida al sexo, piropos groseros, propuestas indecentes.

Violencia de género: cualquier acto de violencia basada en género, que tenga como consecuencia, o que tenga posibilidades de tener como consecuencia, perjuicio o sufrimiento en la salud física, sexual o psicológica de la mujer, incluyendo amenazas de dichos actos, coerción o privaciones arbitrarias de su libertad, tanto si se producen en la vida pública como privada. (Reyes1 2004)

La negligencia infantil o abandono es una falta de responsabilidad parental que ocasiona una omisión ante aquellas necesidades de los niños para su supervivencia y que no son satisfechas temporal o permanentemente por los padres, cuidadores o tutores. Comprende una vigilancia deficiente, descuido, privación de alimentos, incumplimiento de tratamiento médico, impedimento a la educación, etc.

El maltrato emocional es aquel conjunto de manifestaciones crónicas, persistentes y muy destructivas que amenazan el normal desarrollo psicológico del niño. Estas conductas comprenden insultos, desprecios, rechazos, indiferencia, confinamientos, amenazas, en fin, toda clase de hostilidad verbal hacia el niño. Este tipo de maltrato infantil ocasiona que en los primeros años del niño, este no pueda desarrollar adecuadamente el apego y, en los años posteriores, se sienta excluido del ambiente familiar y social, afectando su autoestima y sus habilidades sociales.

Otro tipo de violencia infantil es el Síndrome de Münchausen por poderes, que consiste en inventar una enfermedad en el niño o producirla por la administración de sustancias y medicamentos no prescritos. Generalmente, se trata de un niño en la edad de lactante- preescolar (edad media de 3 años). Los signos y síntomas aparecen solamente en presencia de la madre (habitualmente, el perpetrador del abuso) son de causa inexplicable y los exámenes complementarios no aclaran el diagnóstico. Este síndrome presenta una mortalidad entre 10-20 y su impacto a largo plazo puede dar lugar a desórdenes psicológicos, emocionales y conductuales.

Además, se debe incluir el maltrato prenatal, definido como aquellas circunstancias de vida de la madre, siempre que exista volun- 
tariedad o negligencia, que influyen negativa y patológicamente en el embarazo, parto y repercuten en el feto. Tales como: rechazo del embarazo, falta de control y seguimiento médico del embarazo, negligencia personal en la alimentación e higiene, medicaciones excesivas o no prescritas, consumo de alcohol, drogas y tabaco, exposición a radiaciones y otras. En los últimos tiempos, se habla de maltrato institucional, que consiste en cualquier legislación, programa o procedimiento, ya sea por acción o por omisión, procedente de poderes públicos o privados, por profesionales al amparo de la institución, que vulnere los derechos básicos del menor, con o sin contacto directo con el niño.

Se realizó el análisis estadístico de los casos de violencia en el Ecuador datos obtenidos del Consejo Nacional para la Igualdad intergeneracional CNII, se determinó que, a nivel de país y entre los años 2000 y 2010, "el 44\% de los niños de 5 a 17 años están expuestos casi siempre a castigos 22 violentos (golpes, insultos, encierro) en sus hogares". (Consejo Nacional para la Igualdad Intergeneracional, 2014). 6 de cada 10 mujeres y niñas, de entre 15 a 64 años, han sido víctimas de violencia en algún momento de su vida, es decir 3'260.340. La gran mayoría de los casos no se denuncian; sólo el $10,8 \%$ de todas las mujeres, adolescentes y niñas -víctimas de violencia sexualpresentan la denuncia, mientras el $40 \%$ no dio a conocer el hecho a ninguna persona, esto, debido a que al $28 \%$ de las que avisaron no les creyeron; y, al 16,3\%, les pidieron que no dijeran nada de lo sucedido. (SENPLADES, 2013)

En el año 2012, en las Unidades de Atención de las Familias del MIES se registraron 17.370 atenciones por distintas formas de violencia en contra de niñas, niños y adolescentes, entre ellas, la negligencia $(42,13 \%)$, el maltrato psicológico $(25,3 \%)$ y el maltrato físico (18\%) fueron las más frecuentes. Casos de abuso sexual (7,73\%), violencia intrafamiliar $(5,13 \%)$ y maltrato institucio- nal $(1,66 \%)$ también fueron atendidos. En este registro, Galápagos (14,76\%), Manabí (12,81\%), El Oro (11\%), Azuay $(9,46 \%)$ y Loja $(7,66 \%)$ aparecen como las provincias en las que mayor porcentaje de atenciones se efectuaron. (Consejo, 2017-2021, Agenda nacional para la Igualdad Intergeneracional)

En Ecuador, durante el año 2014, fueron registradas 271 denuncias de delitos sexuales en instituciones educativas. En el año 2013, de las 634 denuncias de violencia sexual contra niñas y adolescentes registradas, el $85 \%$ se relaciona con violaciones (Agencia Pública de Noticias del Ecuador y Suramérica - ANDES, 2014) y, según la Fiscalía General del Estado, solo un 1\% de estas denuncias obtiene sentencia (Fiscalía General del Estado, 2016), siendo que, diariamente, se reciben 3 denuncias de violación a niñas menores de 14 años. Según datos de la Fiscalía General del Estado, de cada 10 víctimas de violencia, 6 son niños, niñas y adolescentes, de las cuales el $80 \%$ son del sexo femenino. (Ministerio, 2017), Protocolos de actuación frente a Situaciones de Violencia detectadas o cometidas en el sistema educativo.

Según un estudio de Unicef, hasta 2015 se conoce que el $30 \%$ de los niños ecuatorianos es reprendido mediante violencia física. Hay otros tipos de castigos: $2 \%$ son mediante tratos degradantes, 3\% mediante insultos y burlas, al $1 \%$ no le hacen caso, al $15 \%$ le privan de gustos y apenas el $41 \%$ de niños tiene padres que prefieren dialogar y buscar ayuda.

Y la violencia contra los niños se conecta con otro tipo de violencia, que es la de género. Según un estudio de la Universidad San Francisco de Quito (USFQ) y Plan Internacional, el $82,5 \%, 8$ de cada 10 madres, de la Sierra considera que las niñas no pueden evitar ser víctimas de violencia sexual. De hecho, el 82,2\% de las madres entrevistadas confesó que no conocía las diferentes formas de denuncia ante un caso de agresión en su entorno.

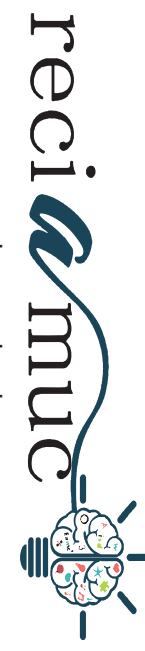


La mayoría de las niñas entrevistadas para este documento dijo que la violencia física y sicológica es más común en la casa y en la escuela, como formas de reprenderlas. La violencia sexual, según el informe, se da en espacios de reuniones o fiestas, fundamentalmente cuando hay consumo de alcohol y cuando las personas encargadas de su cuidado no les prestan atención. Además, todavía hay zonas del país donde se resuelven temas de violencia sexual a través de pagos o intercambio de animales, así como con matrimonios con el agresor. (Ministerio, 2017) , Protocolos de actuaciòn frente a Situaciones de Violencia detectadas o cometidas en el sistema educativo.

En Ecuador el $47 \%$ de los niños, niñas y adolescentes han recibido -por parte de sus progenitores- algún tipo de maltrato físico. De esa cifra, el 48\% vivió castigos extremos (golpes, encierros, baños en agua fría y privación del alimento). Las cifras son de la Encuesta de la Niñez y Adolescencia en el Marco de la Intergeneracionalidad (ENAI). Aunque el levantamiento de la información corresponde a 2016, siete de cada diez niños en el Ecuador son víctimas de maltrato a manos de miembros de su entorno familiar. Así lo establece un estudio de la Universidad Técnica Particular de Loja (UTPL) realizado por el curso de Pedagogía Familiar. El informe confirma que un $75 \%$ de la violencia proviene del padre, madre o de ambos progenitores. (Telégrafo, 2018),

En el Ecuador el $48 \%$ o $50 \%$ de las mujeres son agredidas física, verbal o psicológicamente y se creería que está conducta está ligada a una baja educación o escalas de cultura, pero en realidad no es así. Esta forma de violencia se da en todos los estratos de la sociedad. La violencia es violencia venga de donde venga.

Se han realizado notables investigaciones acerca de la violencia infantil relacionados con el estrés, adaptabilidad sociopsicológica, violencia extrafamiliar, y violencia entre padres.
Los socios de Loandhera diferencian el efecto de la crianza autoritaria y autorizada en el maltrato infantil. Muestran que la crianza autoritaria se asoció con todos los tipos de maltrato infantil, mientras que la crianza autorizada se asoció con un riesgo menor de todos los tipos de maltrato infantil. Ha sido ampliamente aceptado que cualquier tipo de violencia familiar es un factor de riesgo de la victimización de niños. Emeryetal, constantemente encontró que la violencia de pareja era un predictor significativo de polivictimización infantil. Si bien existen asociaciones entre diferentes tipos de violencia dentro de una familia, también se ha demostrado que la polivictimización familiar está asociada con la violencia extrafamiliar como el acoso escolar y el acoso cibernético .Cualquier política y programa de prevención de la violencia debe considerar la multiplicidad de la violencia familiar y cómo impactaría en la seguridad y salud infantil. (Chan, 2019)

Los resultados del cuestionario de la Escala de Abuso Infantil y Trauma (CATS), indican un alto nivel de abuso psicológico y físico de niños en familias. Esto sugiere que muchos padres aplicaron castigos físicos a sus hijos en el proceso de su educación. Los resultados generales muestran que los estudiantes con un alto nivel de violencia doméstica tienen un bajo nivel de adaptabilidad sociopsicológica (menos del 20\%). Mientras que en un grupo con un nivel bajo, el indicador es más del $41 \%$. Este indicador muestra el impacto negativo de la violencia familiar en la adaptación sociopsicológica.

En el factor de adaptación muestra los estudiantes no tienen dificultades para superar los obstáculos de la vida. Se adaptan rápidamente a las condiciones del entorno social y forman un sistema adecuado de relaciones con los objetos sociales sin embargo La baja tasa indica la incapacidad de interactuar efectivamente con otras personas, el deseo de pasar más tiempo solo. La falta de conexiones sociales y aislamiento conduce a la agresión hacia la familia, co- 
nocidos, colegas o transeúntes comunes. (Douglas,2019). Los sujetos con un alto nivel de violencia son más inestables emocionalmente, más sensibles a la angustia y propensos a las emociones negativas en comparación con aquellos con un bajo nivel de violencia. Los estudiantes con un alto nivel de traumas psicológicos, debido a la violencia doméstica, tienen bajos niveles de control subjetivo.

Otros estudios destacan la exposición a la violencia entre padres de niños se ha asociado con comportamientos más agresivos por parte de ellos en varios contextos. La hipótesis de la bidireccionalidad de la violencia en el contexto de la CPV (violencia entre padres) se debe a que los niños que han sufrido abuso de los padres o han observado violencia entre los padres tienden a ser más violentos hacia los padres. En una población comunitaria, la agresión infantil podría representar una respuesta funcional a la tensión familiar o un intento de hacer frente a una educación inadecuada para los padres..(Ibabe et al., 2020)

Los estudios aseguran que la violencia intrafamiliar se produce por distintos factores entre ellos: el machismo, el alcohol, el apego seguro, el estrés y la presión por momentos difíciles de un adulto. Estos factores vinculados a la violencia hacen que coexistan agresores más impacientes; sin embargo, el índice más alto es por el apego inseguro del hombre y de la mujer que no tuvieron una íntima relación, la confianza y los afectos familiares. Estos individuos van buscando el afecto seguro en otras personas y eso hace que sean inestables e intranquilos $\mathrm{y}$, por ende, no puedan brindar una educación segura a sus hijos.

Los estilos de crianza inadecuados ocasionan formas de violencia normalizada por las familias, provocando carencias afectivas en los hijos y tensiones del aprendizaje generalmente de nerviosismo, ansiedad, inseguridad, culpas y estrés. Según la revisión documental realizada se evidencia que las prácticas de crianza que asumen los padres ante la educación de sus hijos se ven condicionada por la personalidad, temperamento, equilibrio emocional, aspectos socioeconómicos, culturales y nivel de instrucción de los cuidadores. La forma más usual de disciplinar a sus hijos se basa en los malos tratos.

\section{Conclusiones}

- Los padres o adultos a cargo son los principales responsables del cuidado, protección y formación de los niños, desde la infancia hasta la adolescencia. Los estilos de crianza son permisivos, negligentes, autoritarios y democráticos. Sin embargo, según factores personales, contextuales, psicológicos se encontró que los estilos de crianza pueden ser: interrumpida, consistente, sensible, insensible, deficiente y disfuncional.

- La violencia infantil ocasiona carencias afectivas y tensiones en el aprendizaje como el nerviosismo, la angustia, temor, ansiedad, inseguridad y el estrés. Las carencias afectivas provocan comportamientos inhibidos o agresivos.

- Los niños, niñas y adolescentes han recibido -por parte de sus progenitoresalgún tipo de maltrato físico, emocional como mecanismos de disciplina y asumiendo la violencia normalizada como el mecanismo de educar a los hijos.

- Los padres o cuidadores necesitan competencias parentales positivas para garantizar hijos educados, con autonomía, e inteligencia emocional para afrontar las adversidades de la sociedad. Las competencias parentales necesarias son las vinculares, formativas, protectoras y reflexivas; sin embargo, los programas de crianza deben considerar el tratamiento de posibles factores psicoemocionales y sociales que presenten los progenitores o quienes están al cuidador del menor. 


\section{Bibliografía}

Arellano, B., Gramszlo, C., \& Woodruff-Borden, J. (2018). Parental reactions to children's negative affect: The moderating role of parental GAD. Journal of Anxiety Disorders, 53(1), 22-29. doi:https:// doi.org/10.1016/j.janxdis.2017.10.006

Bagán, G., \& Tur-Porcar, A. (2019). Learning and Parenting in Spanish Environments:Prosocial Behavior, Aggression, and Self-Concept. Sustainability, 11(1), 1-14. doi:10.3390/su11195193www.mdpi. com/journal/sustainability

Campaert, K., Nocentini , A., \& Menesini , E. (2018). The Role of Poor Parenting and Parental Approval for Children's Moral Disengagement. Journal of Child and Family Studies, 27(1), 2656-2667. Obtenido de https://link.springer.com/article/10.1007/ s10826-018-1097-1

Chan, K. (2019). Child Victimization in the Context of Family Violence. Int. J. Environ. Res. Public Health, 16(1). doi:10.3390/ijerph16193569

Consejo Nacional par la Igualdad. (2018). Agenda Nacional para la Igualdad Intergeneracional. Quito, Ecuador: ANII. Obtenido de https://www.igualdad.gob.ec/agenda-nacional-para-la-igualdad-intergeneracional-2017-2021/

Coulborn Faller, K. (2020). The child sexual abuse disclosure controversy: New perspectives on an abiding problem. Child Abuse Negl, 99(1). doi:10.1016/j.chiabu.2019.104285

Dudek, J., Colasante, T., Zuffianò , A., \& Haley, D. (2020). Changes in Cortical Sensitivity to Infant Facial Cues From Pregnancy to Motherhood Predict Mother-Infant Bonding. Child Development, 91(1), e198-e217. doi:https://doi.org/10.1111/ cdev.13182

Esplugues, J. (2008). ¿Hay violencia justa? Reflexiones sobre la violencia y la justicia basada en los derechos humanos. Daimon Revista Internacional de Filosofia, 43(1), 5-14. Obtenido de http://revistas.um.es/daimon/article/view/96051

Ferreira, S., Magalhães, E., \& Prioste, A. (2020). Social Support and Mental Health of Young People in Residential Care: A Qualitative Study. Anuario de Psicología Jurídica, 30(1), 29-34. doi:https://doi. org/10.5093/apj2019a12

Geo Salud. (2018). Tipos de maltrato infantil.

Gómez Muzzio , E., \& Muñoz Quinteros, M. (2014). Manual Escala de Parentalidad Positiva. Santiago de Chile, Chile: Fundación Ideas para la Infancia.

Guallpa Sinchi, N., \& Loja Cárdenas, B. (2015). Es- tilos de crianza de los padres de estudiantes con bajo rendimiento. Cuenca, Ecuador: Universidad de Cuenca. Obtenido de http://dspace.ucuenca. edu.ec/handle/123456789/21872

Heather, D., \& Rachna, N. (2019). Domestic and family violence, child support and 'the exemption'. Journal of Family Studies, 1-16. doi:https://doi.org/ 10.1080/13229400.2019.1653952

Ibabe, I., Arnoso, A., \& Elgorriaga, E. (2020). Childto-Parent Violence as an Intervening Variable in the Relationship between Inter-Parental Violence Exposure and Dating Violence. Int. J. Environ. Res. Public Health, 17(5), 1514. doi:https://doi. org/10.3390/ijerph17051514

Kohlhoff, J., Hawes, D., \& Mence, M. (2016). Emotion Regulation Strategies and Parenting Practices among Parents of Children With Clinic-Referred Conduct Problems. Parenting, 16(4), 302-319. doi:https://doi.org/10.1080/15295192.2016.11849 42

La Hora. (10 de 02 de 2021). Maltrato infantil: 30\% de los niños es reprendido con violencia física. Quito, Pichincha, Ecuador.

Ling Chan, K. (2019). Child Victimization in the Context of Family Violence. Int. J. Environ. Res. Public Health, 16(19), 3569. doi:https://doi.org/10.3390/ ijerph16193569

Lunkenheimer, E., Lichtwarck-Aschoff, A., Hollenstein, T., \& Kemp, C. (2016). Breaking Down the Coercive Cycle: How Parent and Child Risk Factors Influence Real-Time Variability in Parental Responses to Child Misbehavior. Parenting, 16(4), 237-256. doi:https://doi.org/10.1080/15295192.20 16.1184925

McCarroll, J., Fisher, J., Cozza, S., \& Whalen , R. (2020). Child Maltreatment Fatality Review: Purposes, Processes, Outcomes, and Challenges. Trauma Violence Abuse, 13(1). Obtenido de https:// pubmed.ncbi.nlm.nih.gov/31928207/

Paredes Escobar, M., \& Herrera Cadena, J. (2018). Estilos de crianza y el bullying en adolescentes de 13 a 18 años del Colegio "Ecuatoriano Suizo" en el año lectivo 2017- 2018. Quito, Ecuador: Universidad Central del Ecuador. Obtenido de http://www. dspace.uce.edu.ec/handle/25000/16744

SENPLADES. (2013). Plan Nacional del Buen Vivir. Quito, Ecuador: Republica del Ecuador.

Sherryl H, G., \& Roger, B. (2017). Extending Models of Sensitive Parenting of Infants to Women at Risk for Perinatal Depression. Parenting, 17(1), 30-50. doi:https://doi.org/10.1080/15295192.2017.12621 81 


\section{CITAR ESTE ARTICULO:}

Merchán Gavilánez, M. L., Márquez Allauca, V. M., Yanez Palacios, J. F., \& Estrella Acencio, L. P. (2021). Estilos de crianza ante la violencia infantil. RECIAMUC, 5(1), 416-429. https://doi.org/10.26820/reciamuc/5.(1).ene.2021.416429 